\title{
SMART HEALTH MONITORING SYSTEM UTILIZING INTERNET OF THINGS (IoT) AND ARDUINO
}

\section{Ameyshafida Mihat ${ }^{1}$, Norhashimah Mohd Saad ${ }^{1,}$, Ezreen Farina Shair ${ }^{2}$, Rohana Abdul Rahim ${ }^{3}$ and Achmad Bayhaqi Nasir Aslam ${ }^{4}$}

\author{
${ }^{1}$ Fakulti Teknologi Kejuruteraan Elektrik Dan Elektronik, Universiti Teknikal \\ Malaysia Melaka (UTeM), Jalan Hang Tuah Jaya, 76100, Durian Tunggal, Melaka, \\ Malaysia.
}

${ }^{2}$ Fakulti Kejuruteraan Elektrik, Universiti Teknikal Malaysia Melaka (UTeM), Jalan Hang Tuah Jaya, 76100, Durian Tunggal, Melaka, Malaysia.

${ }^{3}$ Hospital Seri Manjung, Jalan Gapis, 32040 Seri Manjung, Perak, Malaysia

${ }^{4}$ Radiology Department, Medical Faculty of Universitas Brawijaya - Saiful Anwar Hospital, Jl. J. A Suprapto No 2 Malang, Indonesia.

*Corresponding Author's Email: norhashimah@utem.edu.my

Article History: Received January 9, 2022; Revised January 24, 2022;

Accepted January 24, 2022

\begin{abstract}
The use of healthcare monitoring systems in hospitals and other health facilities has grown exponentially, and portable healthcare monitoring systems based on new technologies have emerged as a severe concern in several countries throughout the globe. The emergence of the Internet of Things (IoT) technology has contributed to the progress of healthcare from face-to-face counseling to telemedicine. The project aims to design a reliable health monitoring system utilizing the IoT, Arduino, and Android applications. It can be used to measure body temperature, heart rate, and level of oxygen in the blood in both hospitals and homes. The system is also capable to verify that the transmission of the sensor's data to the host computer is done in real-time. The results show that the system reading is approximately $100 \%$ accurate as of the purchased measuring instruments or actual devices. Therefore, doctors and family members can now monitor and track the patient's health anywhere through this smart health monitoring system.
\end{abstract}

KEYWORDS: IoT, Body Temperature, Heart Rate, Blood's Oxygen Level. 


\subsection{INTRODUCTION}

The Internet of Things (IoT) has connected everything inside during the previous decade, and it has been labeled the next technological revolution [1]. The Internet of Things is most widely used in healthcare management, where it is used to monitor health and environmental factors. The Internet of Things (IoT) is the process of establishing a connection between computers and the internet using sensors and networks. These interlinked components might be utilized in health surveillance systems. Modern technologies now include a customizable interface, personal assistant gadgets, and mental health care to aid individuals in living more intelligent lives [2].

Health monitoring is a significant concern in today's climate. Patients have serious health difficulties as a consequence of an insufficient health monitoring system. The new health monitoring system is unable to deliver real-time patient health warnings and is constrained by the distance between the patient and the hospital [3]. Additionally, it requires time to collect data from patients. It is inefficient in terms of cost. A low-cost contemporary gadget capable of providing real-time data. The primary reason for developing this system is to address the absence of effective health monitoring in community life, the disparity between rural and urban health care centers, and the inability of the present health monitoring system to give real-time patient health alerts [4].

As a result, the usage of in hospitals and other health institutions, healthcare monitoring systems has significantly enlarged, and new technology-enabled portable healthcare monitoring devices have become a serious issue in several nations worldwide [5]. The emergence of the Internet of Things (IoT) technology has contributed to the progress of healthcare from face-to-face counseling to telemedicine. Numerous Internet of Things (IoT) applications are also being developed at the moment.

Thus, doctors and family members may now monitor or follow a patient's health using the Android application [6]. When monitoring, recording, and tracking changes in patient health metrics over time becomes necessary, Internet of Things (IoT) Monitoring comes in helpful [7]. This database of health parameter changes was created using Internet of Things (IoT) health monitoring. This article presents a smart health monitoring system that can monitor patients' fundamental health symptoms in an Internet of Things (IoT) 
environment [8]. This device may be used in both hospitals and households to monitor and record numerous factors such as body temperature and heart rate [9]. This is a large sensor-based project using cutting-edge technologies.

The project aims to create a prototype of a healthcare monitoring device that can detect and wirelessly transmit a human body's temperature, pulse rate, and the level of oxygen in the blood to a host PC. The health-monitoring equipment created here is an Internet-ofThings (IoT) system. It's based on the Arduino UNO. The Arduino UNO is a common prototyping board that is often used in Internet of Things projects. To develop this medical IoT system, the Arduino is utilized to link the pulse LM-35 temperature sensor, MAX30100 pulse oximeter, character LCD, and ESP8266 Wi-Fi modem. The computed pulse rate, temperature, and oxygen saturation level in the blood are shown on the character LCD attached to an Arduino and sent to the cloud platform through a Wi-Fi access point. Regularly, health-related data such as pulse rate, body temperature, and blood oxygen saturation level are updated and recorded to the ThingSpeak platform. The data may be utilized to maintain a patient's medical history.

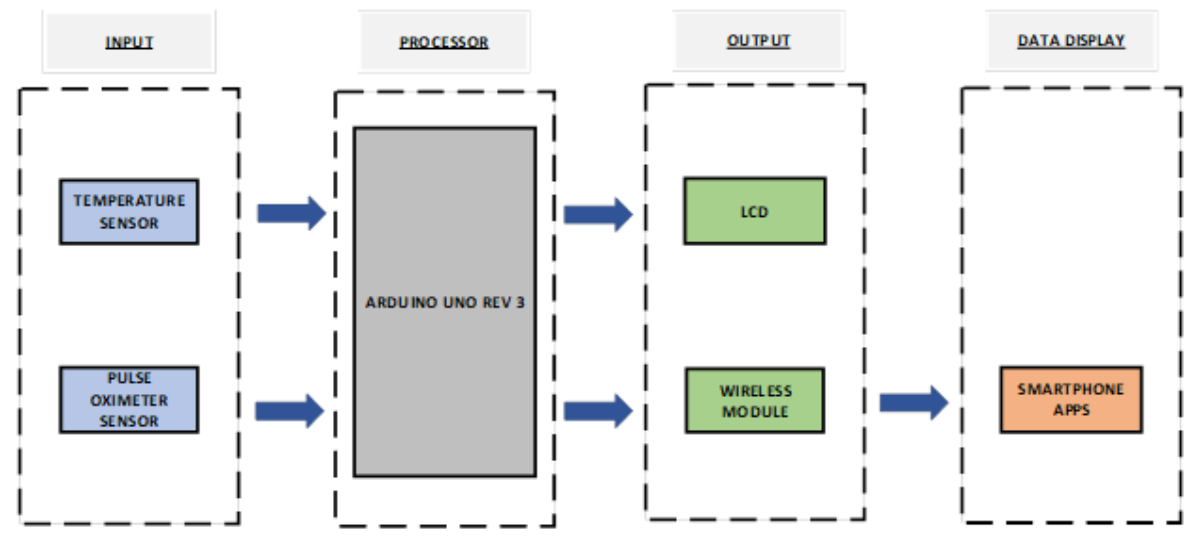

Figure 1: Smart Health Monitoring System Block Diagram

Based on Figure 1, this smart health monitoring system's inputs include a temperature sensor and a pulse oximeter sensor, which detects all of the health indicators that must be monitored. The inputs then communicate the measured parameters to the Arduino UNO, which interacts with the data and then exports it to the outputs. LCD and wireless modules serve as outputs. All parameter data is shown in the smartphone application, ThingSpeak Apps, which enables users to monitor their health status based on the gathered data. 


\subsection{METHODOLOGY}

\subsection{Hardware Design}

For this Smart Health Monitoring System, based on Figure 2, the flowchart displays the whole process of constructing a project system, which includes both hardware and software required to operate the system. The system is initiated when two distinct input signals initialize the body temperature through an LM35, the heart rate through a pulse sensor, and the blood oxygen saturation level through a Max30100 pulse oximeter sensor.

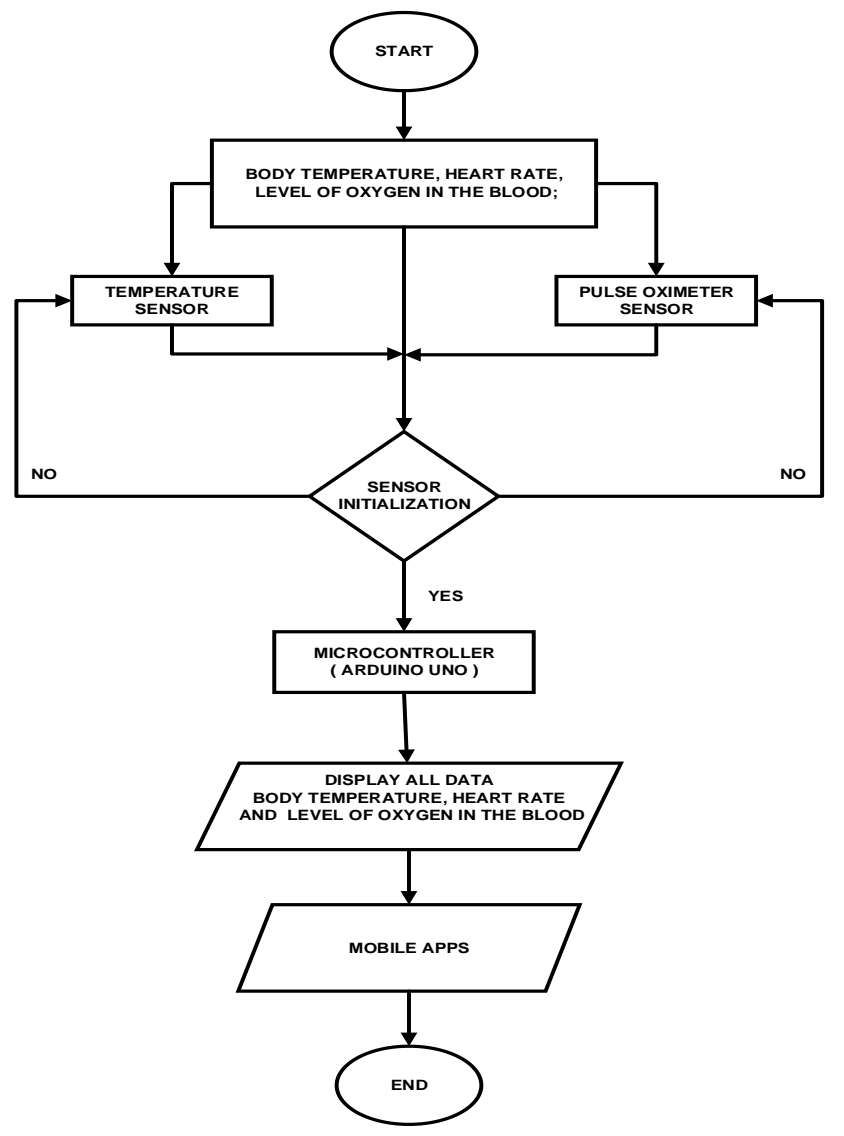

Figure 2: Overall flowchart of project system

Following successful initialization of the input sensor, both input sensors are sent to the microcontroller, which is an Arduino Uno Rev 3. After transferring the input data to the microcontroller (Arduino Uno Rev 3), the Arduino Uno rev 3 will transmit the body temperature, heart rate, and oxygen saturation level in the blood to the LCD as 
output, which will be shown on the LCD screen as an output display. The data is sent in real-time to the WiFi module and then to the mobile application (www.thingspeak.com) for analysis and display. Simultaneous real-time updates of the processed and visible data represent the state of the human body's temperature, heart rate, and blood oxygen saturation level.

The schematic circuit was installed using Proteus software as shown in Figure 3. The Arduino Uno Rev 3 microprocessor acts as the microprocessor in this system that interfaces with all component input and output. These components include an LM35 and MAX30100 Pulse Oximeter sensor. The LM35 sensor is used for body temperature detection and a MAX30100 Pulse Oximeter sensor for heart rate and blood's oxygen level detection. It is located on the MAX30100 Pulse Oximeter board in the schematic as the gauge's initialization point. The body temperature, heart rate, and blood oxygen level are sensed when the fingertips are put on the MAX30100 Pulse Oximeter sensor board. The chip receives the collected data. The heart rate, blood oxygen saturation level, and temperature of the human body are all detected and shown on an LCD screen.

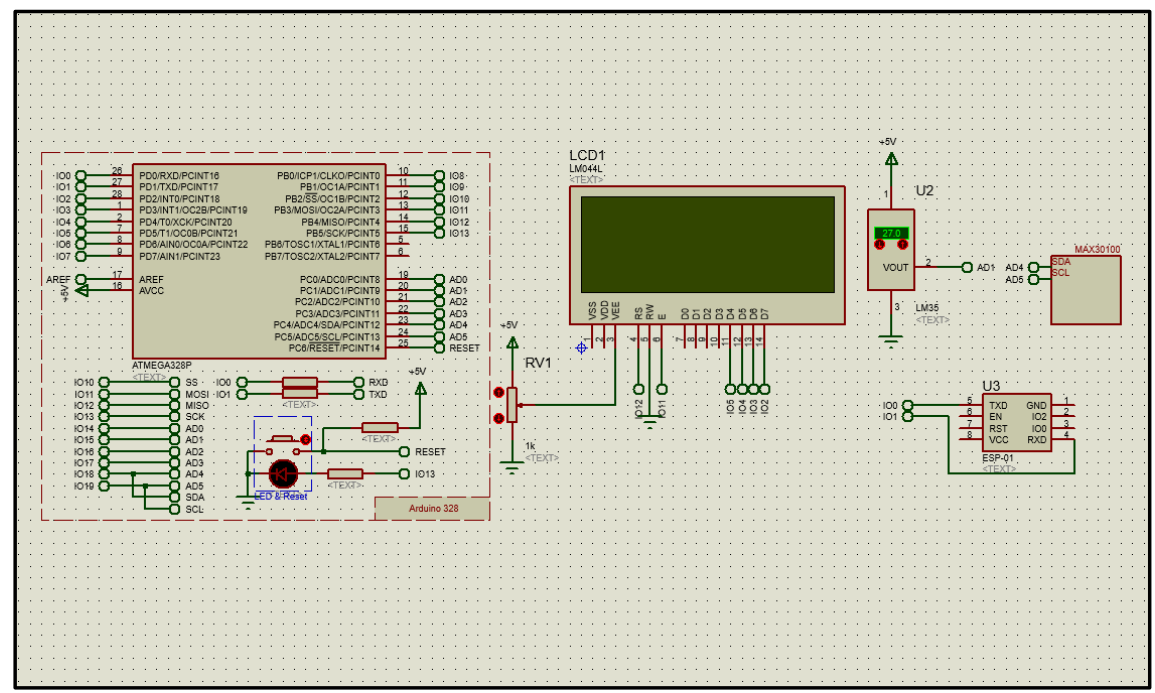

Figure 3: The architecture of a body temperature, heart rate, and blood's oxygen level system is shown schematically.

Based on Figure 4, this Arduino UNO serves as the system's brain in this project. It connects all of the input and output components. It is capable of reading inputs such as a pulse sensor, an LM-35 temperature sensor, and a biosensor and converting them to outputs such as an LCD and the ThingSpeak platform on a mobile phone. 


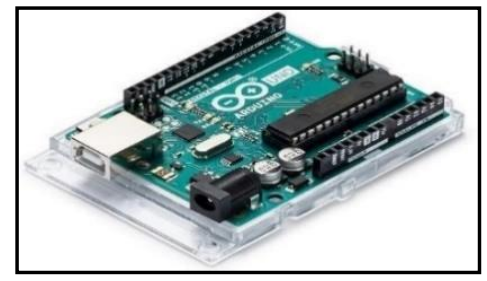

Figure 4: Arduino UNO Rev 3

The LM35 sensor as shown in Figure 5 is used to determine the body temperature in this project. The sensor is positioned against the body and detects the internal temperature of the subject. It is calibrated linearly in degrees Celsius. It has the limited self-healing ability. Additionally, it eliminates the need for external calibration.

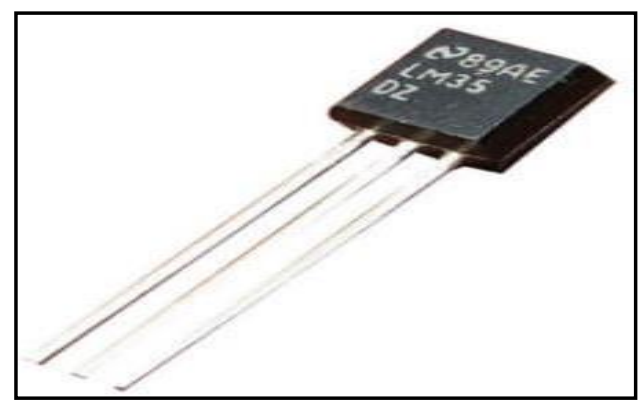

Figure 5: LM35 Temperature Sensor

Based on Figure 6, a pulse oximeter is used to determine the oxygen saturation of a patient's blood noninvasively. Light is transmitted through a transparent, pulsating artery bed, which is often a fingertip or earlobe, using a red and infrared light source, photodetectors, and a probe.

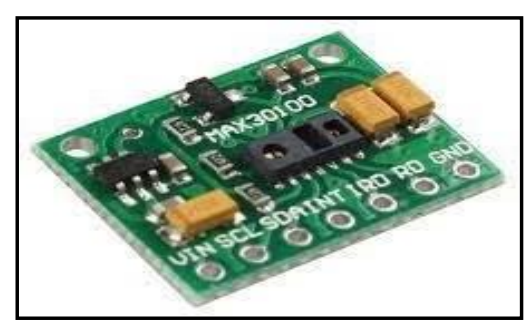

Figure 6: MAX30100 Pulse Oximeter

Figure 7 shows a liquid-crystal display (LCD) is a type of flat panel display, electronic visual display, or video display that uses liquid crystals to regulate light. 20x4 refers to the fact that each of the LCD's four rows will show 20 characters, for a total of 80 characters shown at 
any one moment. Our LCD has a total of 16 pins.

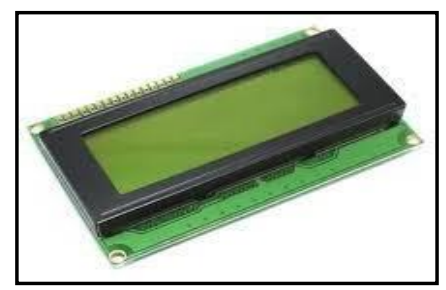

Figure 7: LCD 20X4 Display

NodeMCU as shown in Figure 8 is an open-source development board and firmware. It allows you to program the ESP8266 WiFi module using the Arduino IDE or the simple and powerful LUA programming language. It combines the functions of a WIFI access point and a microcontroller-based station.

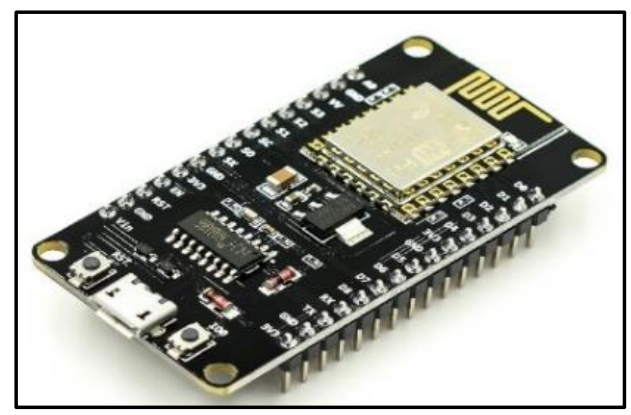

Figure 8: NodeMCU V3 ESP8266 (Wifi Module)

\subsection{Software Design}

This project utilizes a separate Arduino IDE (Integrated Development Environment) for software development. The Arduino IDE as shown in Figure 9 is a free and open-source software development environment for creating and compiling code for Arduino Modules. It is the official Arduino program that simplifies the learning process for non-technical individuals. 


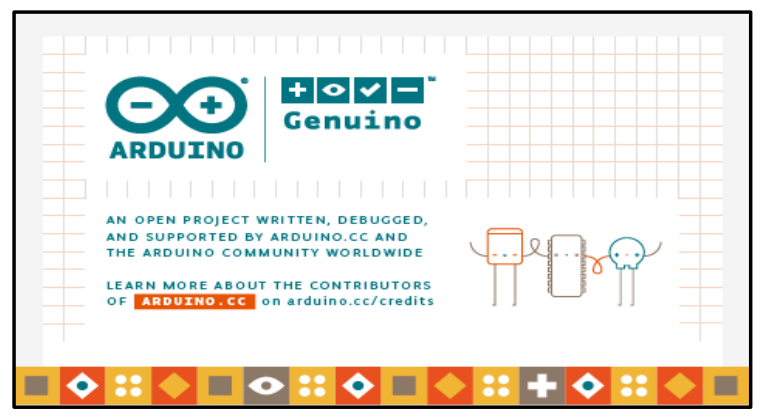

Figure 9: Integrated Development Environment (Arduino IDE)

Proteus Design Suite 8.9 as shown in Figure 10 is used to develop drawings and validate the traceability of project systems. The Proteus Design Suite is a collection of proprietary software tools used mostly for electrical design automation. Electronic design professionals and technicians create electronic schematics and print them for the production of printed circuit boards by using the Proteus software.

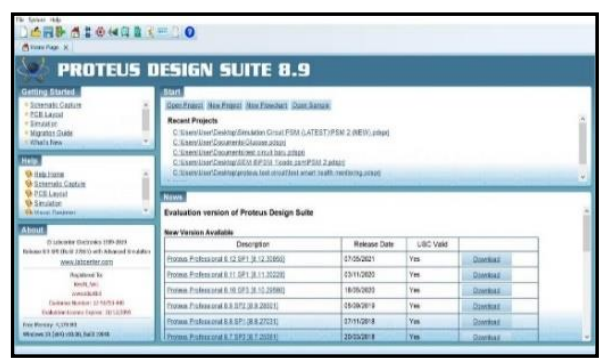

Figure 10: Proteus Design Suite 8.9 Version

Figure 11 shows the Overview of ThingSpeak. ThingSpeak is an open-source Internet of Things platform that enables the storage and retrieval of data from HTTP-enabled devices. ThingSpeak enables users to construct sensor recording applications, location tracking applications, and social networks for things that continuously update their state.

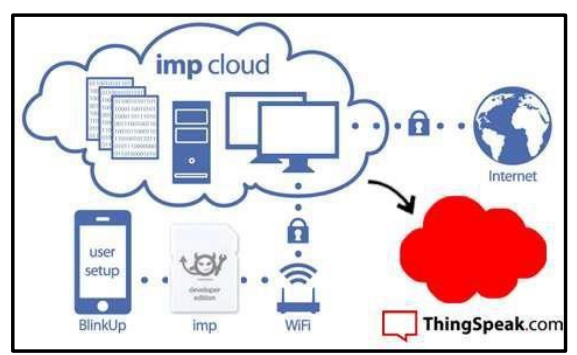

Figure 11: Overview of ThingSpeak (www.thingspeak.com) 


\subsection{RESULTS}

Based on Figure 12, the smart health monitoring with Internet of Things (IoT) and Arduino has been successfully implemented using an Atmega328 microcontroller that uses Arduino Uno Rev 3. This Arduino serves as the primary controller for the project, allowing it to communicate with all of the gear involved. It is capable of measuring and monitoring fundamental human health by using the LM35 sensor, which functions as a body temperature sensor, and the MAX30100 sensor, which functions as an oximeter sensor, which measures heart rate and detects oxygen levels in the blood. Additionally, the Arduino gathers real-time health data via a MAX30100 sensor that measures heart rate, oxygen levels in the blood, and a temperature sensor that is linked to the Arduino.

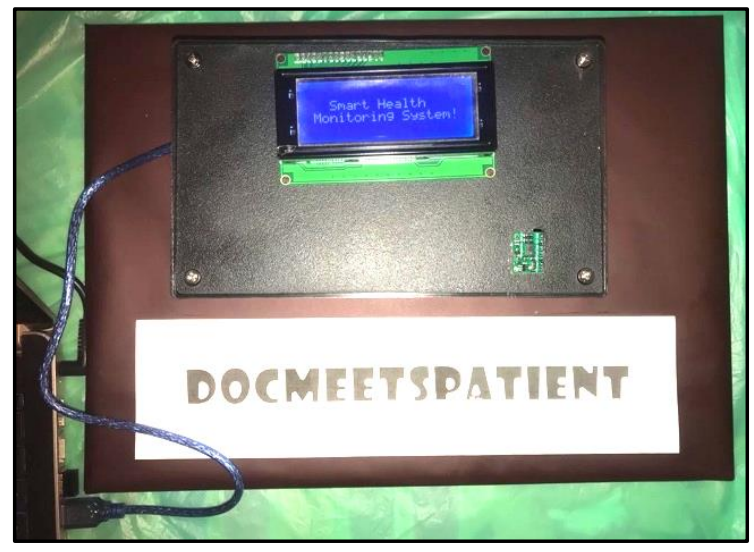

Figure 12: Shows the prototype Smart Health Monitoring System

(DocMeetsPatient)

Apart from that, the Arduino is also responsible for collecting, displaying, and transmitting data to the ESP8266, an IoT module, in this project. The common ESP8266 Internet of Things module is linked to the Arduino through a Universal Uniform Transmitter (UART) Receiver, which is responsible for connecting the circuit to the internet and transmitting health data to an IoT (ThingSpeak) server as shown in Figure 13 (a), (b), and (c). This ThingSpeak application is in charge of storing and monitoring medical data. Furthermore, this circuit is capable of not only transmitting health data to a server but also displaying real-time data on a $20 \times 4$ LCD. This is advantageous for healthcare workers monitoring patients on-site. 


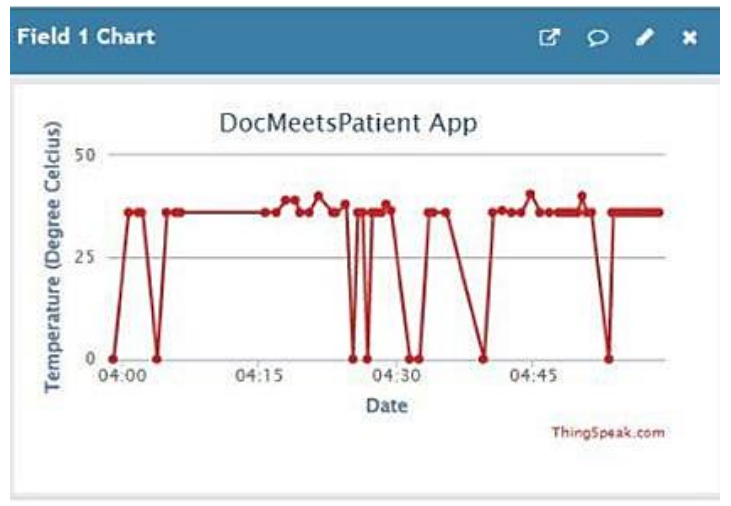

(a)

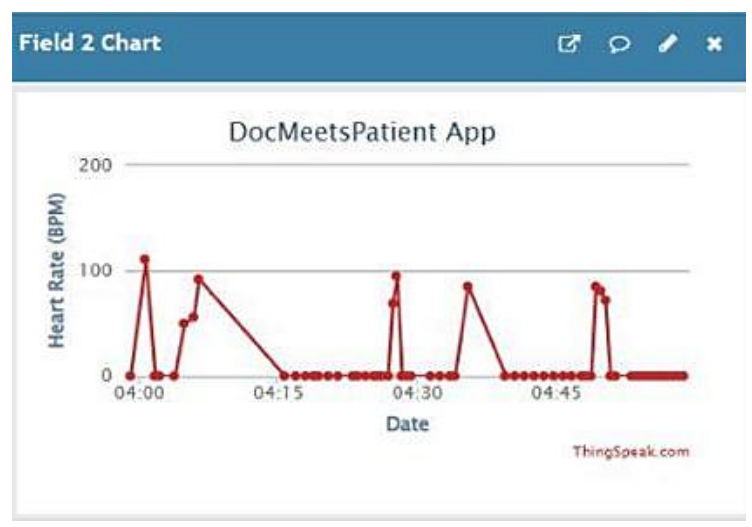

(b)
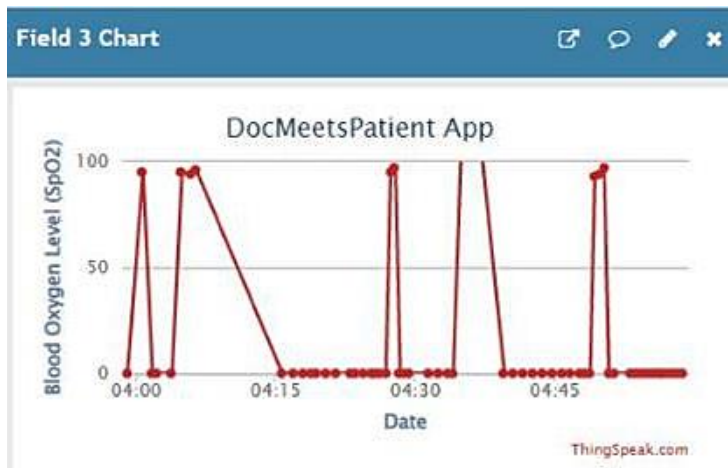

(c)

Figure 13: Shows the successful development of the Internet of Things (IoT) for (a) Body Temperature, (b) Heart rate, and (c) Blood Oxygen Level. 
Health monitoring is performed non-invasively with this method by simply putting a finger on the sensor and detecting it straight through the skin. Simply by putting a finger on the input sensor, the user will be able to immediately measure and monitor their heart rate, body temperature, and oxygen saturation levels in their blood.

\subsection{ANALYSIS}

The Smart Health Monitoring System uses the LM35 body temperature sensor, and the MAX30100 pulse oximeter sensor to detect the heart rate and oxygen levels in the blood. From these experiments, 10 reading values were taken from 10 different people to test the level of accuracy of the sensor readings used as shown in Figure 14, Figure 15, and

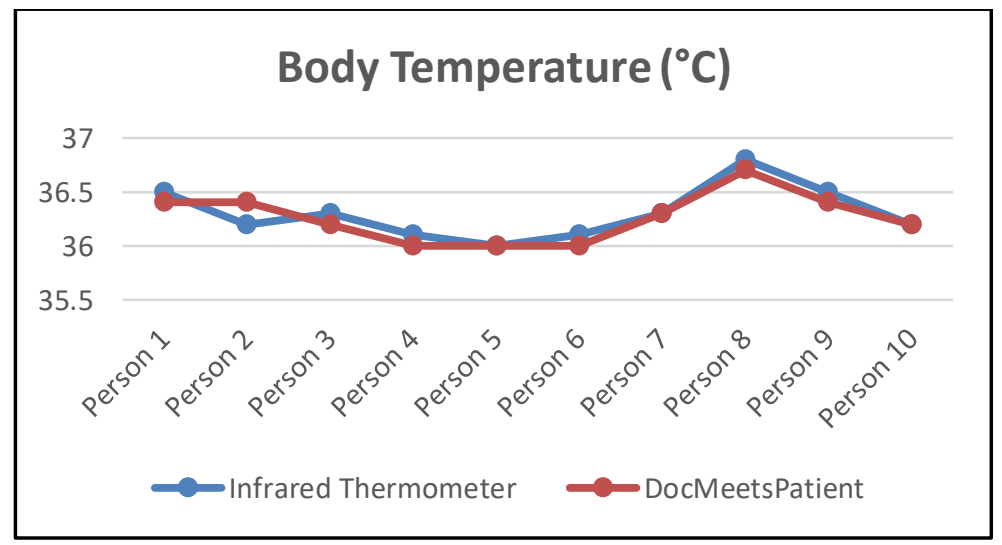

Figure 14: Results of 10 trial sample readings for Body Temperature.

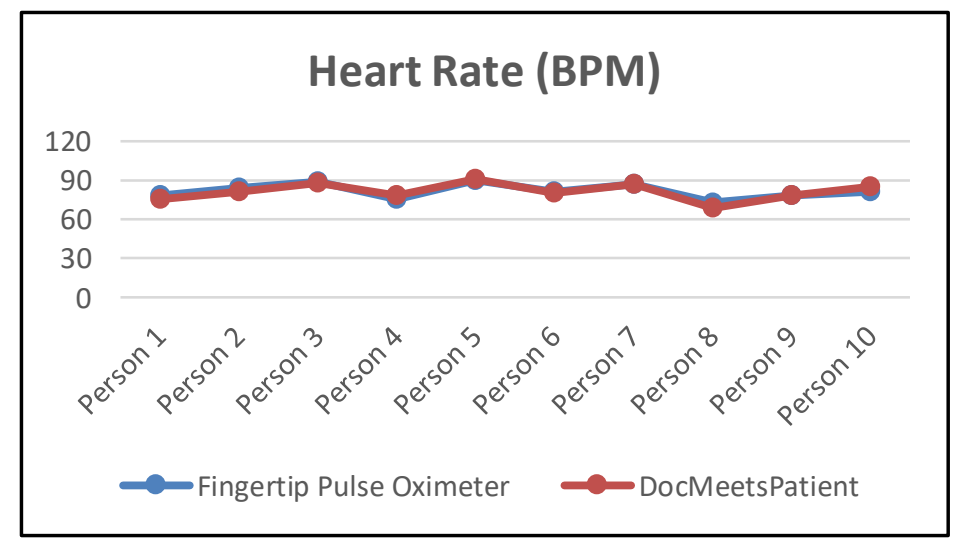

Figure 15: Results of 10 trial sample readings for Heart Rate 


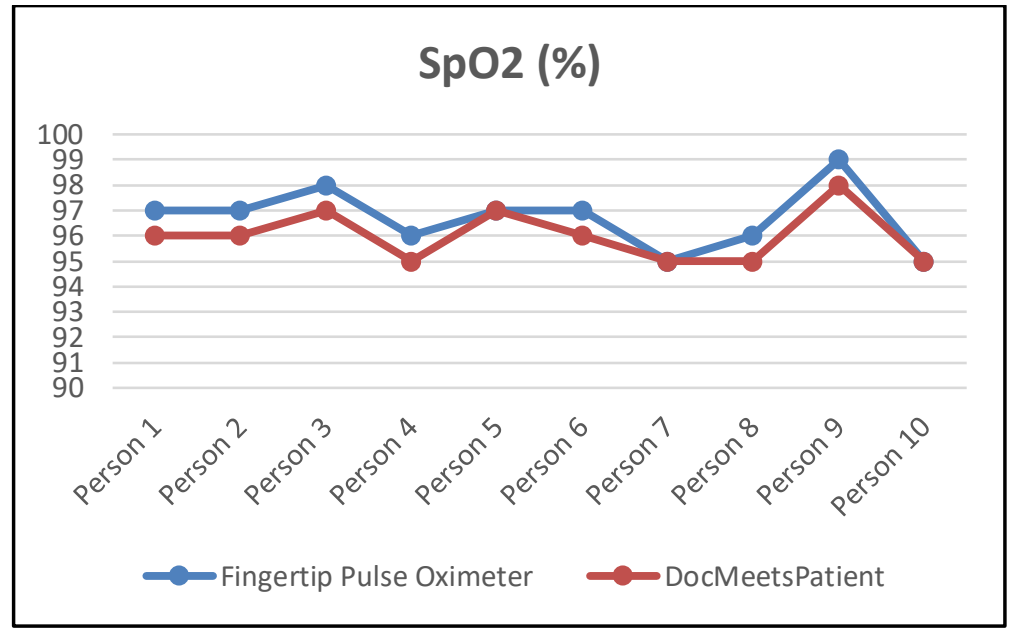

Figure 16: Results of 10 trial sample readings for Blood Oxygen Level

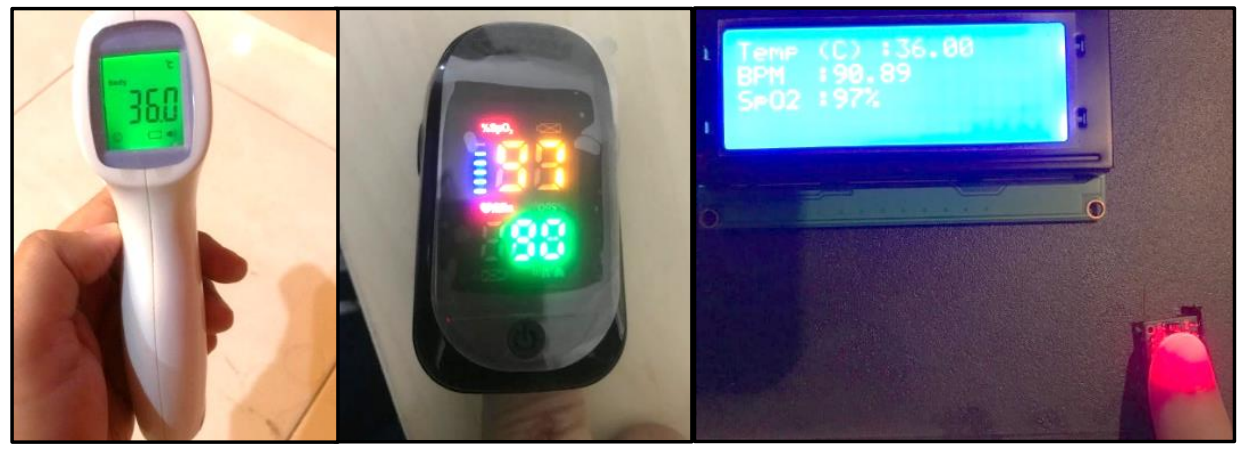

Figure 17: Shows a comparison of body temperature, pulse rate, and blood oxygen levels that have been measured using a real device with readings measured using a designed Smart Health Monitoring System.

Figure 16. Once the testing is carried out, the readings from all these sensors show values that are approximately the same as the purchased measuring instrument (the actual device) as shown in Figure 17. This indicates that the design of a reliable smart health monitoring system has been successfully developed. However, if the finger is not properly placed on the MAX30100 Pulse Oximeter, an erroneous reading may be generated. For pulse rate, only infrared light is needed. Both red light and infrared light are used to measure oxygen levels in the blood. The amount of ambient light hitting the sensor can affect the final value. While using the MAX30100, keep the finger still to avoid an inaccurate reading. Never press the MAX30100 sensor too hard when 
using it. This impairs blood flow, resulting in inaccurate readings. To avoid this, insert the finger gently and keep it still, as this will ensure the most accurate reading.

\subsection{CONCLUSION}

This project is presented to present the construction of a smart health monitoring system by applying the use of Internet of Things (IoT) technology, Android applications, and an Arduino microcontroller. The suggested techniques and approaches operate perfectly and effectively while needing little hardware. The system is suggested to measure a variety of characteristics utilizing sensors such as the LM35 temperature sensor, and the MAX30100 Pulse Oximeter sensor. These sensors are used to detect data on the body temperature, heart rate, and blood oxygen level. By using the IoT platform, health data collected by the three input sensors can be seen on the LCD and in real-time through the ThinkSpeak platform. ThinkSpeak is one example of an IoT platform that will simplify the process of health monitoring on occasion. This developed system provides many facilities for medical staff and families to perform the process of health measurement or monitoring, whether at home or in the hospital.

\subsection{ACKNOWLEDGMENTS}

The authors would like to thank the Faculty of Electrical and Electronic Engineering Technology (FTKEE), Advanced Digital Signal Processing (ADSP) Lab, Fakulti Kejuruteraan Elektrik (FKE), Universiti Teknikal Malaysia Melaka (UTeM), and Ministry of Higher Education (MOHE), Malaysia that supported this research under project FRGS/1/2020/FTKEE CERIA/F00428.

\subsection{REFERENCES}

[1] S. Dharmoji, A. Anigolkar, and P. S. M, "IoT based Patient Health Monitoring using ESP8266," pp. 3619-3624, 2020.

[2] A. Selvanayakam, A. C. Varishnee, M. Kalaivani, and G. Ranjithkumar, "Health Monitoring System Using IoT," Lect. Notes Electr. Eng., vol. 626, pp. 739-750, 2020, doi: 10.1007/978-981-152256-7_68.

[3] A. Rahaman, M. M. Islam, M. R. Islam, M. S. Sadi, and S. 
Nooruddin, "Developing iot based smart health monitoring systems: A review," Rev. d'Intelligence Artif., vol. 33, no. 6, pp. 435-440, 2019, doi: 10.18280/ria.330605.

[4] T. Khan and A. Chakrabarty, "Development of Application based Health Monitoring System using GSM module Anika Tasniem 12101081 Nura Jamil 12101007," 2016.

[5] C. Senthamilarasi, J. J. Rani, B. Vidhya, and H. Aritha, "A Smart Patient Health Monitoring System Using Iot," Int. J. Pure Appl. Math., vol. 119, no. 16, pp. 59-70, 2018.

[6] A. N. A. Yusuf, F. Y. Zulkifli, and I. W. Mustika, "Development of Monitoring and Health Service Information System to Support Smart Health on Android Platform," 4th Int. Conf. Nano Electron. Res. Educ. Towar. Adv. Imaging Sci. Creat. ICNERE 2018, pp. 38, 2019, doi: 10.1109/ICNERE.2018.8642592.

[7] M. T. A. Mahgoub, O. O. Khalifa, K. A. Sidek, and S. Khan, "Health monitoring system using Pulse Oximeter with remote alert," Proc. - 2015 Int. Conf. Comput. Control. Networking, Electron. Embed. Syst. Eng. ICCNEEE 2015, pp. 357-361, 2016, doi: 10.1109/ICCNEEE.2015.7381391.

[8] L. J. Ramírez López, A. Rodriguez Garcia, and G. Puerta Aponte, "Internet of things in healthcare monitoring to enhance acquisition performance of respiratory disorder sensors," Int. J. Distrib. Sens. Networks, vol. 15, no. 5, 2019, doi: 10.1177/1550147719847127.

[9] E. A. Azman and I. Of, "Final year project report title heartbeat and temperature sensor monitor via mobile phone," 2019. 